\title{
Biometric Identification by Fingerprint Image Based Minutiae Detection
}

\author{
Israa Mohamed Khidher \\ Department of Computer Science / Collage of Education \\ University of Mosul
}

Received
$1 . / 1, / 200 V$

Accepted

. $r / 0 r / 200 \wedge$

\section{الخلاصة}

تعد الوثوقية الثخصية تطبيق مهم من تطبيقات الحوار بين الإنسان والحاسبة والتي

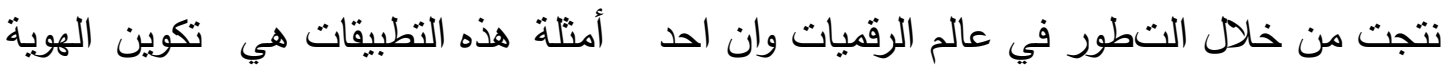
الثخصية ـ يعرف علم المقاييس الثخصية بأنه علم تحديد الصفات الثخصية من خلال المقاييس الفسيو لوجية (الوظائفية ) والصفات السلوكية ـ نو فر المقاييس الثخصية مثل بصمة

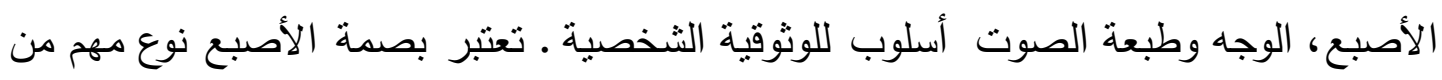
أنواع المقاييس الثخصية التي من المكن استخدامها في التطبيقات المدنية والقانونية.

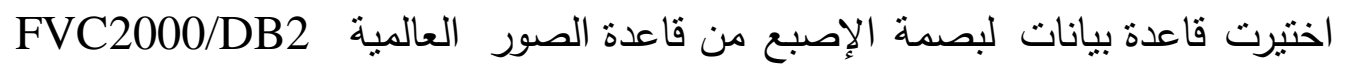

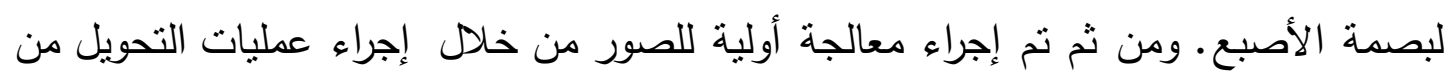

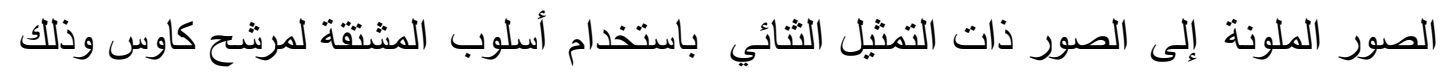

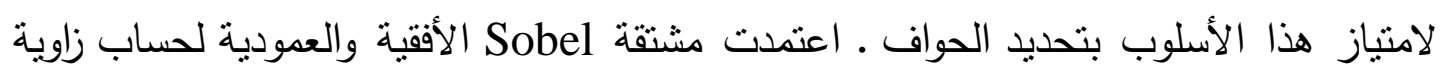

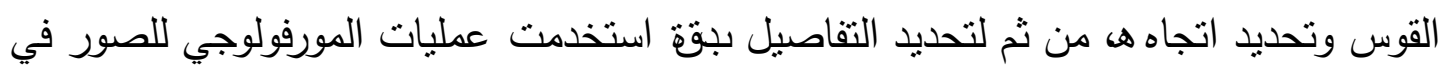
عملية التتحيف ـ قطعت هذه الصور بشكل مقاطع صورية ممثلة بمربعات لفصل خلفية الصورة

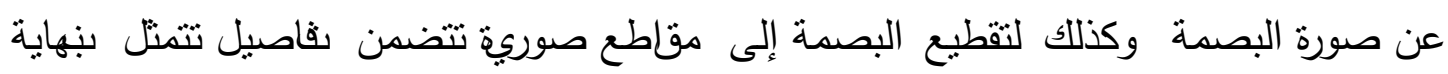

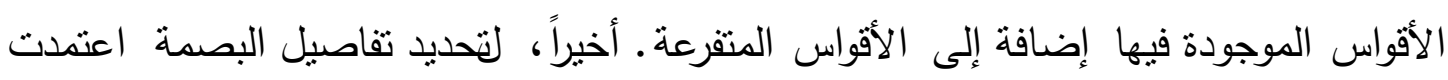

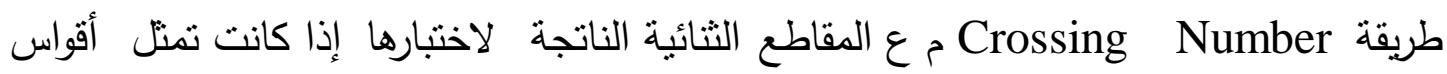

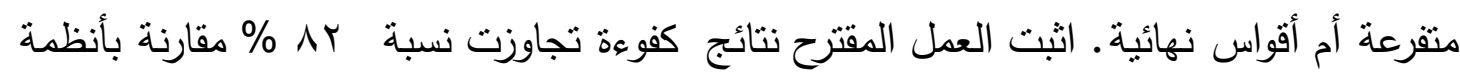
أخرى في نفس المجال.

\section{Abstract}

In an increasingly digital world, reliable personal authentication has become an important human computer interface application, some 
examples where establishing a person's identity. Biometrics is the science of verifying the identity of an individual through physiological measurements or behavioral attributes. Biometrics such as fingerprint, face and voice print offers means of reliable personal authentication. Fingerprints were one of the important forms of biometric identification to be used for law and civilian applications.

Data base images for fingerprint images are selected from the First International Fingerprint Verification Competition FVC2000/DB2. Then a primary processing to these images are performed through the transformation from RGB color to binary form using the derivative of Gaussian filter scheme because, the excellence of this scheme in edge detection. The Sobel horizontal and vertical gradient are relied to compute the ridge angle and to detect its orientation, then to detect the details accurately image morphological operations are used for thinning operation. These images are segmented to image portions represented by blocks also to segment the finger tip to image portions contain details assimilate by ridges ending as well ass ridges bifurcation. Indeed, to detect the details of the fingerprint Crossing Number method is relied with the resultant binary portions to test them if they represent ridge ending or ridge bifurcation. The proposed work shows efficient results exceeds $82 \%$ compared with other system on the same field.

\section{Introduction}

Accurate automatic Biometric identification is an important field to the operation of our increasingly automated information society [3]. Biometrics is defined as "reorganization of a person by determining the authenticity of a specific physiological and/ or characteristic posse by the person" [9].

Fingerprint identification has become the most important than other characteristics such as face, retina, iris, voice and handgeometry biometric due to its university, performance and accuracy [3][4]. Fingerprint identification has been started since eighteen century by (Edward Henry), who establishes the "Henry system" for fingerprint classification. The progress of this field was continued by the early 20 century. Various fingerprint identification systems that based on fingerprint classification, matching and detection were developed. These systems were used in forensic agenesis as well as civilian applications [9][2].

This rapid increase in fingerprint detection requires feature extraction algorithm. These features are minutia that includes the ridge and bifurcation [4][8]. Automatic fingerprint identification systems (AFIS) are a class of biometric information systems widely used based on minutiae matching. Another system was based on segmenting the 
fingerprint image into ridge zone and background. Statistical analysis is used such as moment other system used three zone such as ridge, valley and background with histogram. Also Fourier and Gabor filter to enhance the fingerprint images before any segmentation [11][10].

The proposed work use RGB fingerprint images then these images are transformed to gray-scale images. The gray-level images in turn are converted to binary images to facilitate minutiae extraction. Thinning process is performed to the fingerprint binary images. The thinned images are segmented into blocks to isolates the foreground from background. Also to extract minutiae features locally per-block. Finally minutiae features such as ridge and bifurcation were extracted based on blocks.

\section{Fingerprint features overview}

Fingerprint represents the image of skin of finger tip. Fingerprint included ridge (black line) separated by valleys [3]. The type of ridge such has ridge ending, bifurcation, crossover represents the local features and known as minutiae used in fingerprint analysis. Commonly different 150 type of minutiae were recognized and defined a sample is shown in figure (1-a). The most important ones are ridge and ridge bifurcation used commonly in fingerprint identification as shown in figure (1-b) [11].

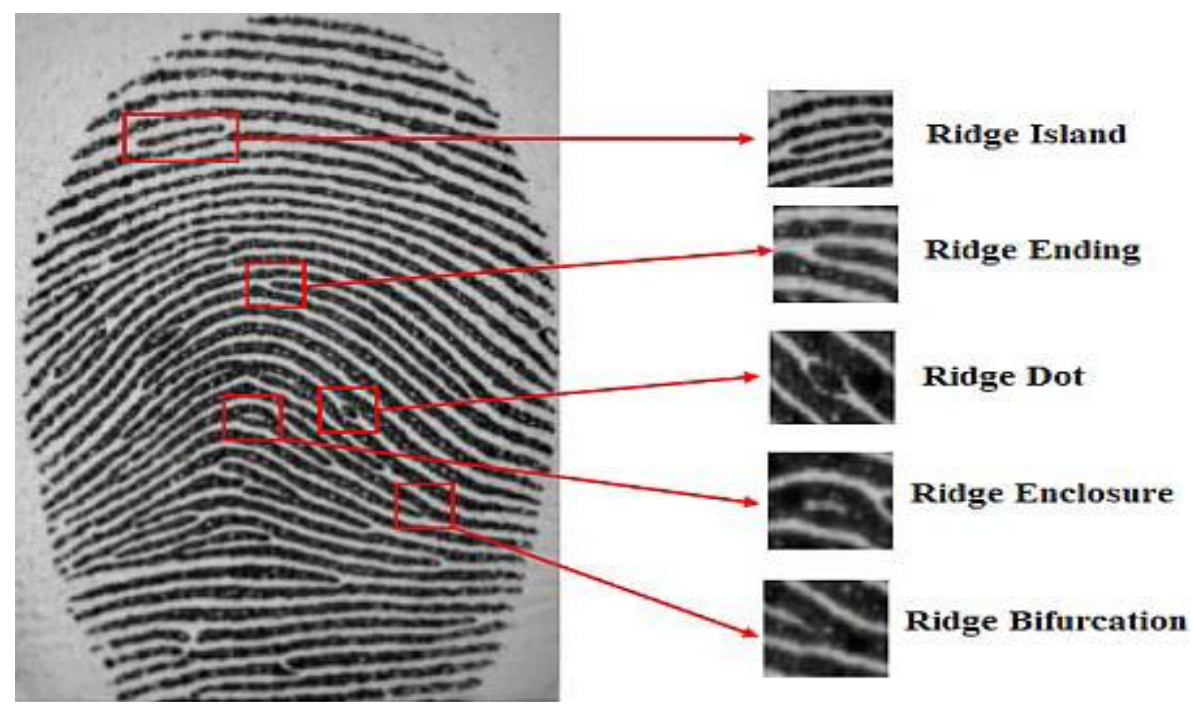

(a)

(b)

Figure (1): fingerprint overview

(a): Different minutiae type

(b): Ridge ending and bifurcation

Ridge ending are the points were the ridge curve terminates, and bifurcation were the ridge split from a single path into two paths as Y-junction as shown in figure (2-a) and figure (2-b) respectively [1]. 

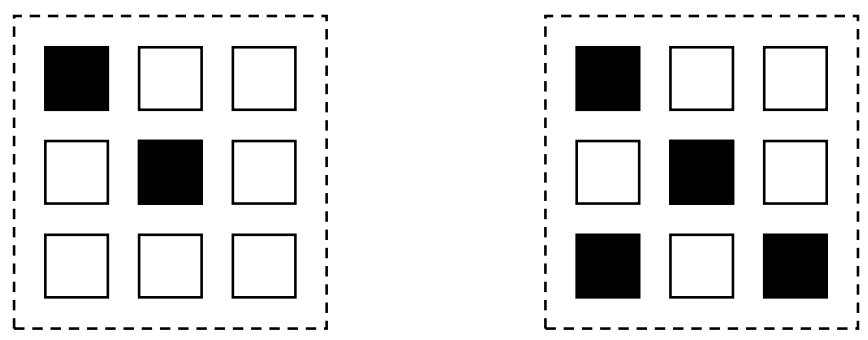

Figure (2): minutiae pixel representation

(a): Ridge ending pixels

(b): Ridge bifurcation pixel

\section{The proposed work}

A sample of 33 color fingerprint data set images are applied as a visual database in this work. These images are selected from FVC2000/DB2 figure (3) shows some images from the sample. The proposed work differs from others work in two points. The first one is that the proposed work uses the block-wise operation instead of pixelwise scheme which is widely used in fingerprint analysis. The second is that, the segmentation process is performed not only to isolate background and foreground but also to segment the fingerprint tip as blocks with details while others isolate the foreground and background only.
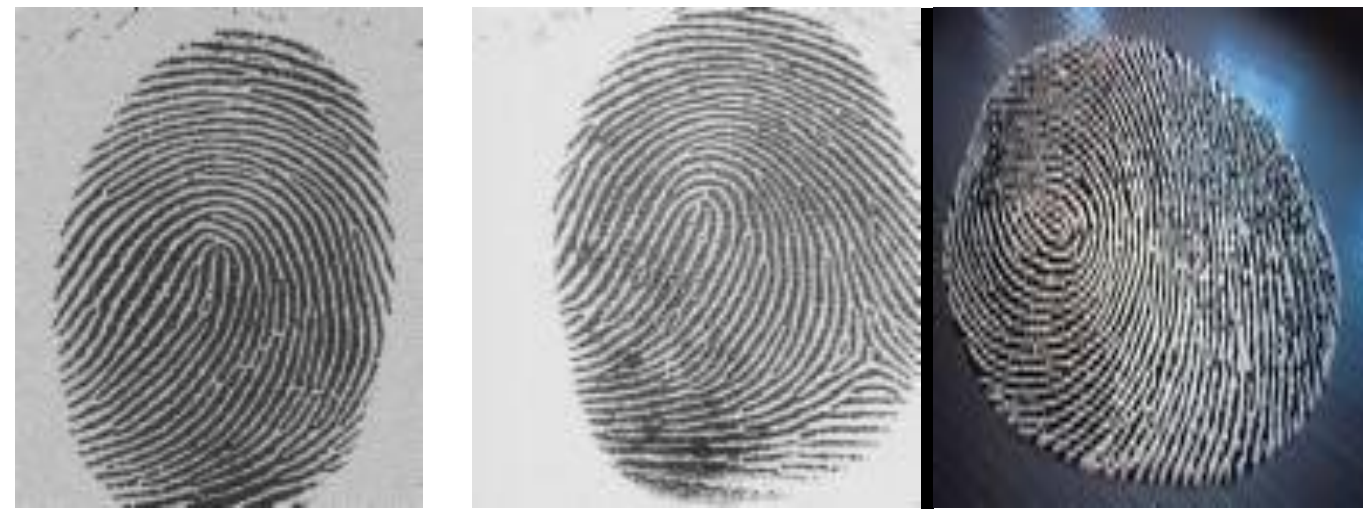

Figure (3): sample from FVC2000/DB2

In order to extract Minutiae features easily and clearly these RGB images are converted to the gray-scale images then to binary images. The gray-scale images is regarded as an intermediate step, because there is no command that directly converts the RGB images to the Binary images. Minutiae detection algorithm is described briefly in the following steps:

1) Pixel orientation.

2) Image binarization

3) Thinning

4) Segmentation

5) Minutiae detection. 
These steps are shown in figure(4). A detailed description to each step is discussed in the follows:

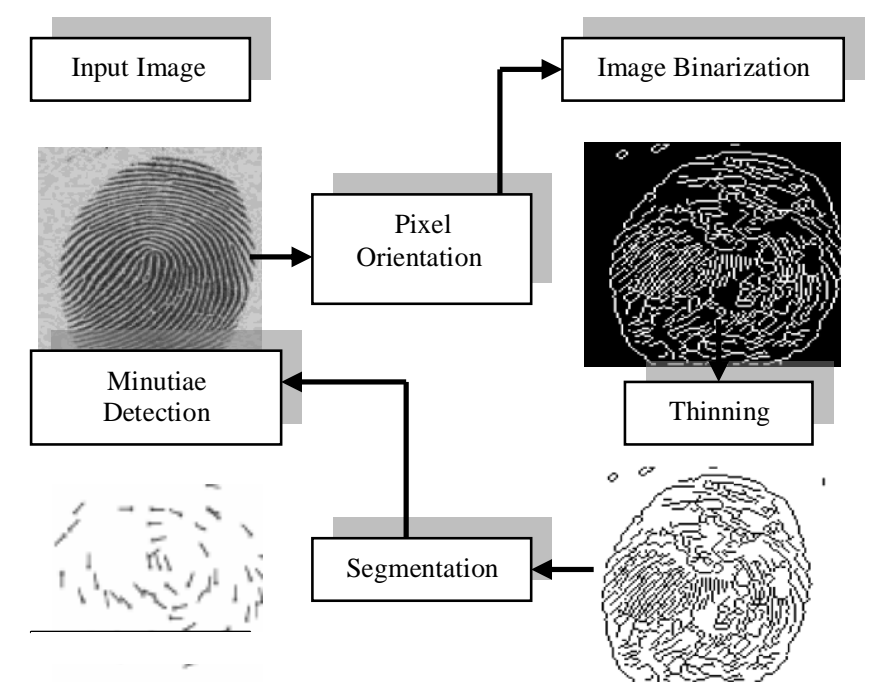

Figure (4): minutiae detection algorithm

\subsection{Pixel Orientation Computation}

The orientation field in fingerprint image defines the local angle of the ridge contained in fingerprint. It is an essential step in fingerprint analysis. These orientations are estimated in block-wise scheme and its computation is performed as follows:

1) A block of $3 \times 3$ is centered at pixel $(i, j)$ which represent the angle of the associated block.

2) The gradient $d x(i, j)$ and $d y(i, j)$ for each pixel in the block are computed that represents the gradient magnitude in $\mathrm{x}$ (horizontal) and $y$ (vertical) directions [10]. This computation is done using Sobel gradient operators. The horizontal mask sobel operator

$$
\mathrm{Sh}=\left(\begin{array}{ccc}
1 & 0 & -1 \\
2 & 0 & -2 \\
1 & 0 & -1
\end{array}\right)
$$

And the vertical mask sobel operator

$$
\mathrm{Sv}=\left(\begin{array}{lll}
1 & 2 & 1 \\
0 & 0 & 0 \\
1 & 2 & 1
\end{array}\right)
$$

The angle of the block is estimated by equation (1) [7].

$$
\theta(i, j)=\tan ^{-1} \frac{d y}{d x}
$$




\subsection{Binarization}

It is a process that converts a gray-level image into a binary image [4]. This process enhances the contrast between the (ridge and bifurcation) best than other attributes in fingerprint image as shown in figure (5-a) and (5-b). This process facilitates the extraction of minutiae. We apply canny method to detect the strong and weak edges. This is performed by calculating the gradient of derivative of a Gaussian filter. The advantage of canny method than others like prewiett, Robert is it is robust to noise and it is powerful in edge detection.

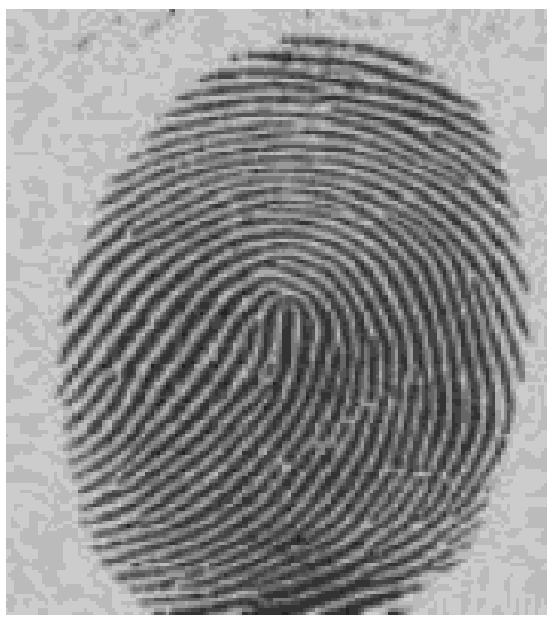

(a)

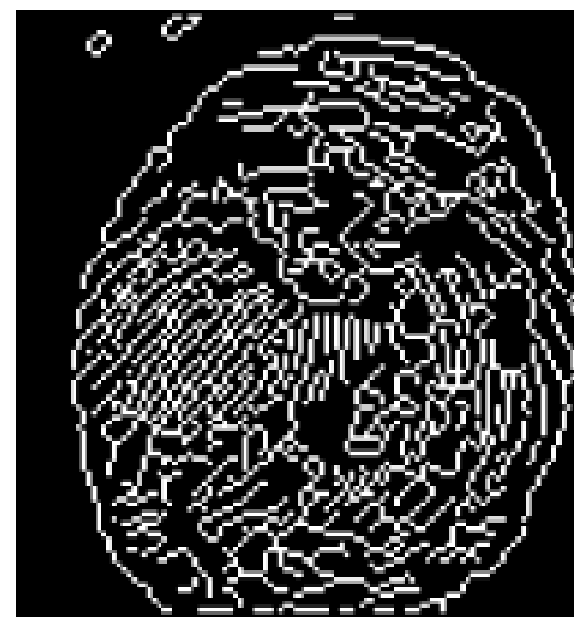

(b)

Figure (5): Binarization Process

(a): Original image

(b): Binarization image

\subsection{Thinning}

The last step in fingerprint image enhancement is the thinning process [3][7]. The binary image is thinning by a morphological operation to simplify minutiae extraction this process erodes the foreground pixels until they become a single pixel in width, but bifurcation that is wider than one pixel. The behavior of the thinning morphological operators is determined by a structuring element. The binary structuring elements used for thinning are of the extended type described under the hit-and-miss transform (they can contain both ones and zeros).

The thinning operation is related to the hit-and-miss transform and can be expressed quite simply in terms of it. The thinning of an image A by a structuring element B is described in equation (2) and (3), where the subtraction is a logical subtraction [7].

$$
\begin{aligned}
& A \otimes B=A-(A * B) \\
&\left.=A \cap(A * B)^{c}\right) \\
& A \otimes\{B\}=\left(\left(\ldots\left(\left(A \otimes B^{1}\right) \otimes B^{2}\right) \ldots\right) \otimes B^{n}\right) \\
&\{B\}=\left\{B^{l}, B^{2}, \ldots, B^{n}\right\}
\end{aligned}
$$


The same thinning algorithm principle is applied in proposed work, by iteratively use the thinning morphological operation. The thinning operation is calculated by comparing the structure element origin with the image pixels. If the foreground and background pixels in the structuring element exactly match foreground and background pixels in the image, then the image pixel associate with the origin of the structuring element is set to background (zero). Otherwise it is left unchanged. This process repeatedly examines each pixel in the image and it is connectivity. It checks whether the neighbor pixel could be detected or not. It continues until no more pixels could be detected.

\subsection{Segmentation}

The segmentation is the process of dividing the image into several regions with same attributes [10]. As known the details of fingerprint are found in ridge (foreground), while the background contains no useful information so it is discarded. In order to extract minutiae the gray image is segmented to blocks of $40 \times 40$ with $3 \times 3$ pixels per block. The mean per block is used for segmentation as in equation (3) [7]:

$$
\mu(k)=\frac{1}{N} \sum_{i=j=1}^{3} P(i, j) \quad k=1,2, \ldots, 40
$$

Where $\mu$ represents mean, $N$ equals the number of pixels in the block and $P$ is the image pixel with it is coordinates ifor row and $j$ for column,

Since there is large mean of intensity in regions of ridges, opposite to uniform background. The mean principle is that the less mean value means the dark region with no details(background) and vise versa. If the value of mean is greater than determined threshold $\mathrm{T}$. The block is considered to be in ridge region, otherwise it is regarded as background so that the block is discarded from next calculation.

\subsection{Minutiae Detection}

In this section the details of minutiae detection is discussed. The Crossing Number $(\mathrm{CN})$ method is used [1]. This method uses a $3 \times 3$ thinned image window produced from previous step. We use the center pixel within a block and examine if it represents minutiae. Also to examine the local neighborhood pixels if they represent a ridge ending or bifurcation. The $\mathrm{CN}$ formula is given in equation (4).

$\mathrm{CN}=0.5 \sum_{i=1}^{8}\left|p_{i}-p_{i+1}\right|$

Pixel $\mathrm{P}$ is the center of the window, its 8 neighbor pixels are scanned clock wise as follows:

\begin{tabular}{|l|l|l|}
\hline 2 & 3 & 4 \\
\hline 1 & $\mathrm{P}$ & 5 \\
\hline 8 & 7 & 6 \\
\hline
\end{tabular}

After $\mathrm{CN}$ calculation the ridge pixel can be classified to only a 2 categories. The first $\mathrm{CN}=1$ that corresponds to ridge ending as shown in figure (2-a). The second is $\mathrm{CN}=3$ which correspond to bifurcation as shown in figure (2-b). Other values of $\mathrm{CN}$ are excluded. 
For every minutia the following information is recorded:

1- Block position in the image.

2- Orientation of the block associated with ridge.

3- Type of minutiae.

Finally, the fingerprint image is displayed with the minutiae detection.

\section{Experimental Results}

This work aim in to detect and analyses the fingerprint images. These are done selecting 33 fingerprint images from FVC2000/DB2.

The results of the proposed work are described as values shown in table (1) the Minutiae includes ridge ending and bifurcation associated with each fingerprint image. Also the results are shown as images in figure (6-a) and (6-b).

Table (1): the results of the proposed work

\begin{tabular}{|c|c|c|c|}
\hline File Name & Ridge ending & Bifurcation & Minutiae \\
\hline T1.jpg & 30 & 33 & 63 \\
\hline T2.jpg & 38 & 78 & 116 \\
\hline T3.jpg & 6 & 114 & 120 \\
\hline T4.jpg & 20 & 85 & 105 \\
\hline T5.jpg & 52 & 51 & 103 \\
\hline T6.jpg & 29 & 52 & 83 \\
\hline T7.jpg & 27 & 68 & 95 \\
\hline T8.jpg & 44 & 66 & 110 \\
\hline T9.jpg & 34 & 120 & 154 \\
\hline T10,jpg & 10 & 98 & 108 \\
\hline T11.jpg & 29 & 104 & 133 \\
\hline T12.jpg & 22 & 90 & 112 \\
\hline T13.jpg & 35 & 120 & 155 \\
\hline T14.jpg & 30 & 74 & 104 \\
\hline T15,jpg & 25 & 84 & 109 \\
\hline T16.jpg & 30 & 84 & 114 \\
\hline T17.jpg & 18 & 61 & 79 \\
\hline T18.jpg & 17 & 74 & 91 \\
\hline T19.jpg & 26 & 68 & 94 \\
\hline T20.jpg & 15 & 104 & 119 \\
\hline T21.jpg & 23 & 61 & 84 \\
\hline T22.jpgp & 23 & 61 & 84 \\
\hline T23.jpg & 27 & 95 & 122 \\
\hline T24.jpg & 21 & 100 & 121 \\
\hline T25.jpg & 26 & 57 & 83 \\
\hline T26.jpg & 33 & 52 & 85 \\
\hline T27.jpg & 19 & 54 & 73 \\
\hline T28.jpg & 19 & 58 & 77 \\
\hline T29.jpg & 25 & 137 & 162 \\
\hline T30.jpg & 16 & 91 & 107 \\
\hline T31.jpg & 43 & 164 & 207 \\
\hline T32.jpg & 41 & 116 & 157 \\
\hline T33.jpg & 30 & 43 & 73 \\
\hline
\end{tabular}


original image

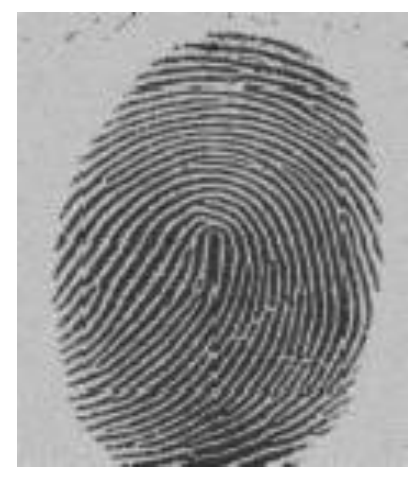

binarized image

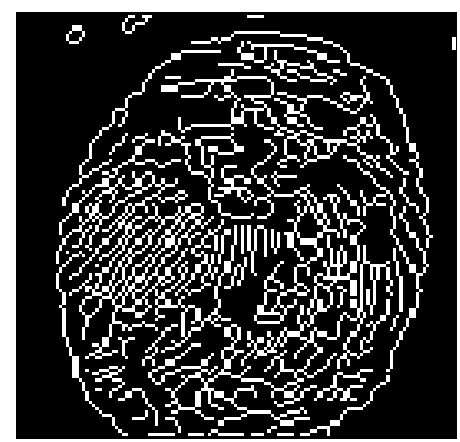

\section{resized image}

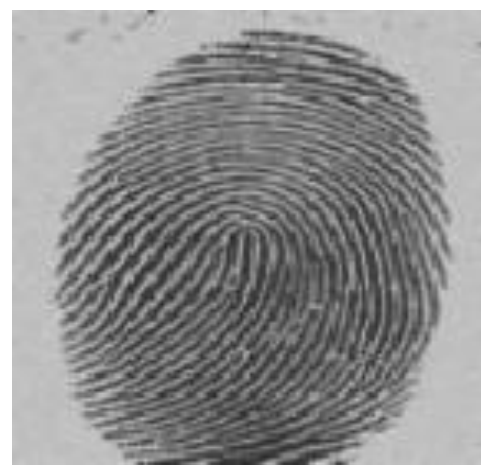

ridge and bifurcation image

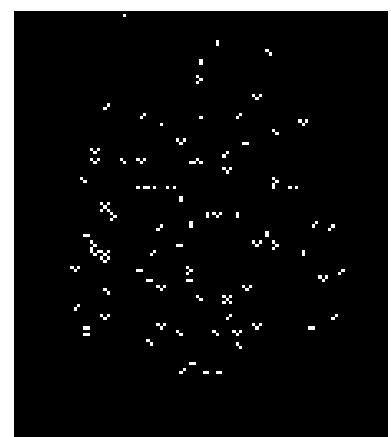

(a)

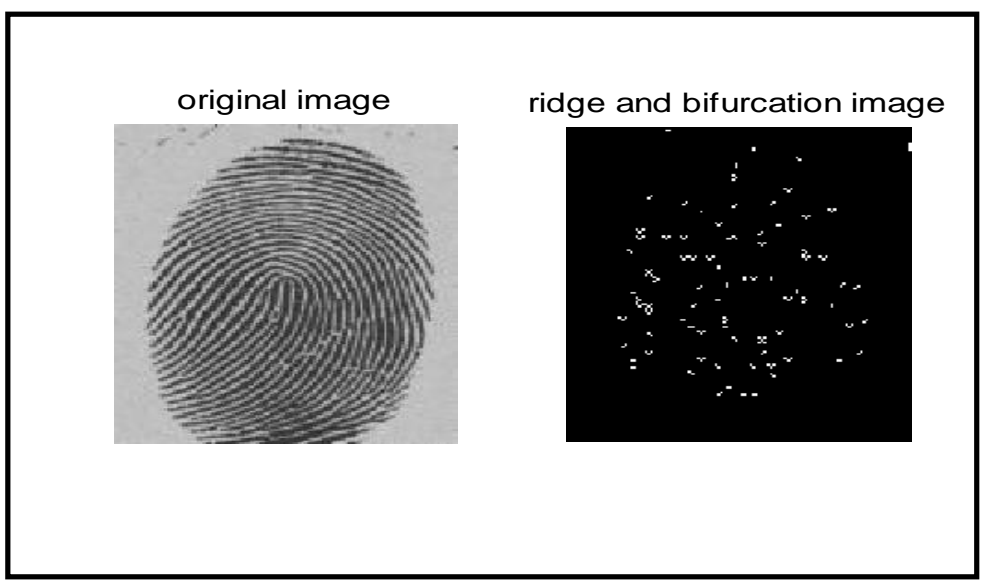

(b)

Figure (6): the results of the system

(a): The steps of the proposed work

(b): First and final step of the system 


\section{Results Analysis}

In order to measure the enhancement of the proposed work. The sensitivity and specificity were measured over the entire database [11]. These measures indicate the ability of the algorithm to detect the true minutiae and reject false minutiae respectively as given in equation (4) and (5) respectively:

Sensitivity $=1-\frac{\text { Missed minutiae }}{\text { Ground truth number of minutiae }}$

Specificity $=1-\frac{\text { False minutiae }}{\text { Ground truth number of minutiae }}$

These measures required a computation of the ground truth value of the database images. This value needs some automatically calculations offered by the system, a selected randomly 10 images from the 33 images are shown in Table 2. Where $M$ represents number of minutiae determined by the expert, $N$ is the number of automatically extracted minutiae, $P$ is the number of correct minutiae, $I$ is number of missing minutiae that could not be recognized by the system and $L$ is the number of false minutiae.

Table 2: Comparison of number of minutiae before and after applying proposed method.

\begin{tabular}{|c|c|c|c|c|c|}
\hline File name & $\boldsymbol{M}$ & $\boldsymbol{N}$ & $\boldsymbol{P}$ & $\boldsymbol{I}$ & $\boldsymbol{L}$ \\
\hline T1.jpg & 63 & 73 & 55 & 8 & 18 \\
\hline T2.jpg & 116 & 114 & 98 & 18 & 16 \\
\hline T3.jpg & 120 & 135 & 117 & 3 & 18 \\
\hline T4.jpg & 105 & 119 & 100 & 5 & 19 \\
\hline T5.jpg & 103 & 102 & 100 & 3 & 2 \\
\hline T6.jpg & 83 & 81 & 77 & 6 & 4 \\
\hline T7.jpg & 95 & 94 & 92 & 3 & 2 \\
\hline T8.jpg & 110 & 111 & 105 & 5 & 6 \\
\hline T9.jpg & 154 & 149 & 140 & 14 & 9 \\
\hline T10.jpg & 108 & 127 & 97 & 11 & 30 \\
\hline
\end{tabular}

The ground truth minutiae for these images were determined automatically as shown in figure (7). This figure shows the minutiae distributions for three sets. These sets are automatically extracted minutiae extracted from the images, number of matching minutiae in the images, and the number of missed minutiae. We see that in this data set images, the ground truth is equal 60 . 


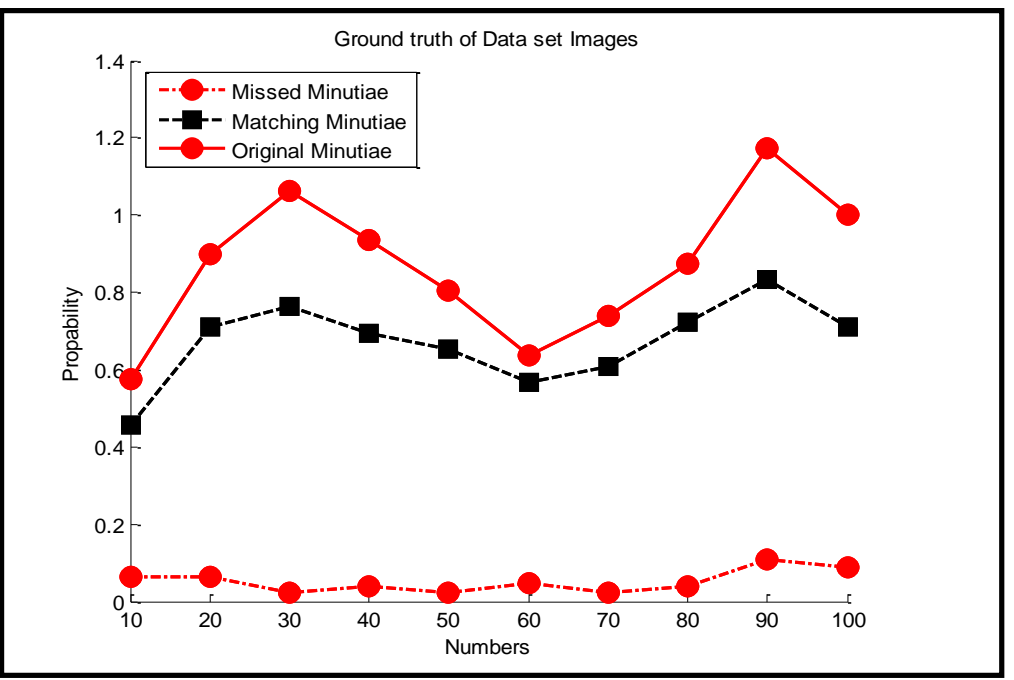

Figure (7): the ground truth of dataset image

We compared our performance of the proposed work with another related work titled "A systematic approach for feature extraction in Fingerprint Images" [5]. We found that the number of true positives due to the proposed methods exceeds the number of true positives found by that approach as shown in table(3).

Table (3): Performance Evaluation over data set image

\begin{tabular}{|c|c|c|c|c|}
\hline Value & \multicolumn{2}{|c|}{ Proposed work } & \multicolumn{2}{c|}{ Systematic work } \\
\hline & Sensitivity & Specificity & Sensitivity & Specificity \\
\hline Mean Value & 82 & 75 & 79.40 & 85.29 \\
\hline Std Deviation & 17.27 & 21.11 & 7.86 & 12.91 \\
\hline
\end{tabular}

\section{Conclusion and future work}

We have proposed a bioinformatics system through the minutiae detection for biometrics verification. The difference between the procedures we proposed than others is that we segment the thinned image, while the others segment the gray-scale image. Also minutiae are the foreground based block-wise scheme is indicated as a region of interest(ROI). The block-wise is performed faster than others method that used pixel-wise scheme. The reliability of the detection depends on the analysis used.

Future work we will try to use HSV color spaces. Also this system will be used for matching with other fingerprint images and with frequency multiresolution. Finally an idea of online minutiae detection with fingerprint sensor connected with the computer will be applied. 


\section{References}

1) Afsar F. A., Arif M. and Hussain M., (2004), "fingerprint Identification and Verification System using Minutiae Maching", in Proceedings of International Conference of Emerging Technologies, Islamabad, Pakistan.

2) Bishnu A., Bhow mick P., Dey J., Bhattacharya B. B., Kundu M. K. and Murthy A., (2002), "Combinatorial Classification of Pixels for Ridge Extraction in a Gray-scale Fingerprint Image", in Proceedings of the third Indian Conference on Computer vision, Graphics \& Image Processing, Ahmadabad, India.

3) Brankica M. opovic and Ljiljana Maskovic, (2007), "Fingerprint Minutiae Filtering Based on Multiscale Directional Information", Electronic Engineering, 20(2): 233-244.

4) Chikkerur Sharat, Govindaraju Venu, Pankanti Sharath, Bolle Ruud and Ratha Nalini, (2005), "Novel approaches for Minutiae Verification in Fingerprint Images", in Proceedings of 7'th IEEE on Application of Computer Vision, Vol.(1):111-116.

5) Chikkerur Sharat, Wu Chaohang and Govindaraju Nenu, (2004), "A Systematic Approach for Feature Extraction in Fingerprint Images", in Proceedings of the First International Conference of Biometric Authentication, China.

6) First International Fingerprint Verification Competition visual database http://bias.csr.unibo.it/fvc2000 [Internet].

7) Gonzalez Rafael C. and Richard E. Woods, (2002), "Digital Image Processing". $2^{\text {nd }}$ Ed., Prentice-Hall, New Jersey, USA, P:793.

8) Liang Xuefeng, Bishnu Arijit and Tetsuo Asano, (2004), "Combinatorial Approach to fingerprint Analysis using Euclidean Distance Transform", Proceedings of IEEE Transactions on Pattern Analysis and Machine Intelligence (PAMI).

9) Maltoni D., Maio D., Jain A. K. and Prabhakar S., (2003), "Handbook of Fingerprint Recognition", Springer, New York, USA.

10) Mih aliescu Preda, Krzysztof Mieloch and Axel Munk, (2007), "Entracer-Entropy sensitive fingerprint feature extraction", University of Gottingen, Germany.

11) Sharat S. Chikkerur, (2005), "Online Fingerprint Verification System", A thesis submitted to the Faculty of the Graduate School of the State University of New York, Department of Electrical Engineering, USA. 